\title{
Putting Order Into the Cloud: Object-Oriented UML-based Enforcement for Document and Application Organization
}

\author{
Doron Drusinsky, James Bret Michael, Thomas W. Otani and Man-Tak Shing \\ Computer Science Department \\ Naval Postgraduate School \\ Monterey, CA, USA \\ \{ddrusins, bmichael, twotani, shing\}@nps.edu
}

\begin{abstract}
In order to improve the ability of users to share data within and across organizations, enterprises can move their data from user-managed devices to data centers accessible via cloud computing services. Such a transition in data storage, access, and management raises technical issues that are not addressed by current operating system, tagging, and configuration and control management technologies. In this paper we describe the notion of a cloud control environment in which cloud services automatically enforce multi-schema-based rules on the organization and manipulation of data objects.
\end{abstract}

Keywords: cloud computing, content management, UML, design pattern.

\section{Introduction}

People typically organize their electronic personal and business documents in an ad hoc manner. Consider for example a person who files his or her personal bank statements in folders, with each folder containing subfolders labeled by year, each of which contains two subfolders named checking and savings, with twelve checking and twelve savings bank statement PDF files in each, respectively. Given the ad hoc organization of the documents, the person must rely on his or her memory to maintain the logical (semantic) relationships among these documents. Such an approach does not scale up for large organizations such as the Department of Defense (DoD) with approximately 3.5 million users of unclassified nontactical information systems who need the ability to share documents via the cloud.

This paper presents a technique for the specification and enforcement of relationships between document objects as well as relationships between document objects and the different applications using software engineering based object-oriented (OO) modeling [1], what we refer to as cloud control (CC). The remainder of this paper is organized as follows. Section 2 presents a financialdocument example and discusses the adaption of the classical $\mathrm{OO}$ principles to organize document objects and applications in the cloud. Section 3 discusses the enforcement of the document-organization schema on instances of actual documents and applications. Section 4 describes the automation support needed. Section 5 discusses related work and Section 6 presents the conclusion.

\section{Object-Oriented Document And Application Organization}

\subsection{A Financial Documents Example}

Consider the financial document organization example depicted in Figure 1.

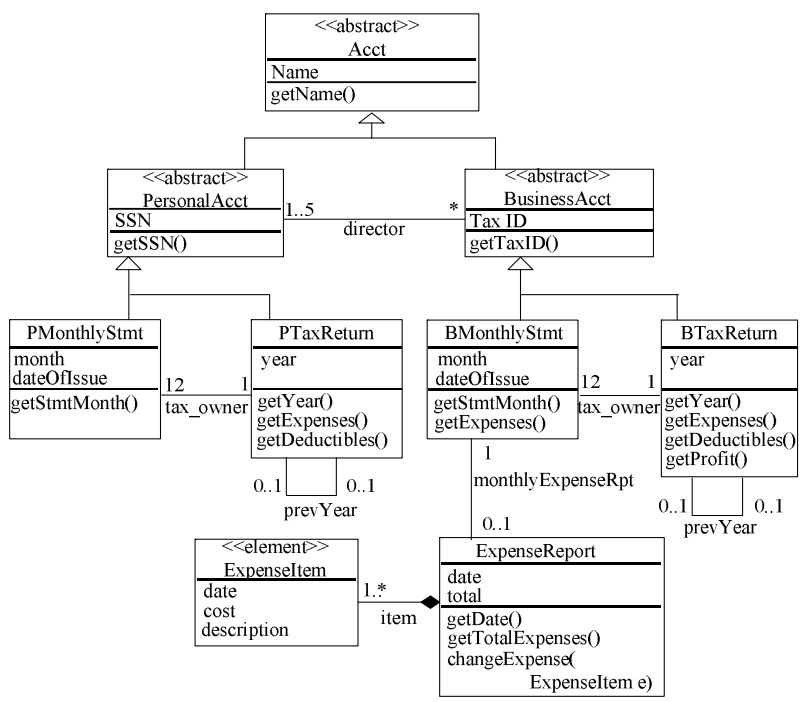

Figure 1. Class diagram schema for banking

It consists of three abstract classes, Account, PersonalAcct and BusinessAcct. Being abstract means, in our context, that no document object exists for these classes. For example, from the document point of view, there is no explicit file or folder object representation for a bank account on the file system; information about bank accounts (e.g., name and SSN that are defined in the Account and PersonalAcct classes respectively and inherited into the PMonthlyStmt and PTaxReturn classes) are captured in the files corresponding to the monthly statements and tax returns instead. There are two kinds of accounts, personal accounts and business accounts. A business account is related to one to five personal accounts of the business directors, and a personal account can be associated with any number of business accounts. A personal account contains two different types of documents 
(PMonthlyStmt and PTaxReturn) while a business account contains three different types of documents (BMonthlyStmt, BTaxReturn, and ExpenseReport). An expense report must be associated with a monthly statement for a business account and each monthly statement must be associated with a tax return document for either personal or business accounts. An expense report consists of a plurality of expense elements, where an element is a part of a document object. Like an abstract class, no document object exists for an element class; an element object contains data that are elements of some other objects. A tax return document may be associated with the tax return document of the previous year. In addition to these relationships, classes contain properties and methods, such as BMonthlyStmt class containing a getExpenses() method.

The UML diagram of Figure 1 is but a visual depiction of a schema. (Refer to [2] for its XML schema counterpart.) Document and application objects that satisfy a schema are called aggregate documents. As with programming counterparts, the cloud can contain a large plurality of aggregate documents that satisfy a given schema. Clearly, this empowers organizations to formally specify document ordering schemas rather than relying on ad hoc end-user organization instances, or relying on the end user to comply with and manually implement some written specification of a mandated document organization schema.

To this end, documents must contain clearly marked property and association segments. Contemporary document objects often contain such information: they are often Extensible Markup Language (XML) documents whose elements are effectively properties, and they often contain links to other documents, for example using a Uniform Resource Identifier (URI) or Uniform Resource Locator (URL) format. Associations can be implemented as embedded URIs or as external information stored by the $\mathrm{CC}$ environment discussed in Section 4. Absent in contemporary document objects however is a specification of the applications that are permitted to operate on those object types and the specific properties that can be read from and written to.

\subsection{Adaptation of Classical OO Principles to Document Objects and Applications}

The three defining properties of classical OO programming (OOP) are inheritance, encapsulation, and polymorphism. In this section we examine their adaptation to the world of document objects and applications.

In OOP, inheritance is a way to form new classes (instances of which are called objects) using classes that have already been defined. Inheritance provides for reusing existing code with little or no modification. In our context, document objects may enjoy the inheritance principle in a similar manner. Consider a cloud repository with two types of documents, checking document (CheckingDoc) and savings document (SavingsDoc). They are both special types of a banking document (BankingDoc), as depicted in the class diagram of Figure 2. The BankingDoc parent has properties for the owner's name, date of creation, and account status. Given that most present-day documents are stored in XML format, one can expect to locate those properties in the corresponding files; for instance, a BankingDoc has an XML element or elements that pertain to the abovementioned properties. Hence, a SavingsDoc virtually contains a name, date of creation, and status although these properties are not present in the SavingsDoc file; rather, their existence is deduced using the inheritance relationship. Note that we might expect to find a mismatch between the namespaces used by documents and those used by the schema, this issue should be resolved by the discovery step discussed in Section 3.

In the most general case, all child documents (i.e., checking and savings type documents) will therefore virtually contain property fields exposed by a parent (UML exposure uses a classifier such as protected or public) although they do not exist verbatim in the respective files. In such a situation, when applying a changeStatus application to a checking document, it changes the status property of its parent banking document. A private qualification in the parent is also useful in our OO cloud interpretation, meaning that no application that is applied to a child document can access the respective parent property field.

Child documents can obviously have their own fields and corresponding applications. For example, a savings document in Figure 2 is specified to allow a readAsSavings application, one that we envision highlights all information that pertains to the interestRate property.

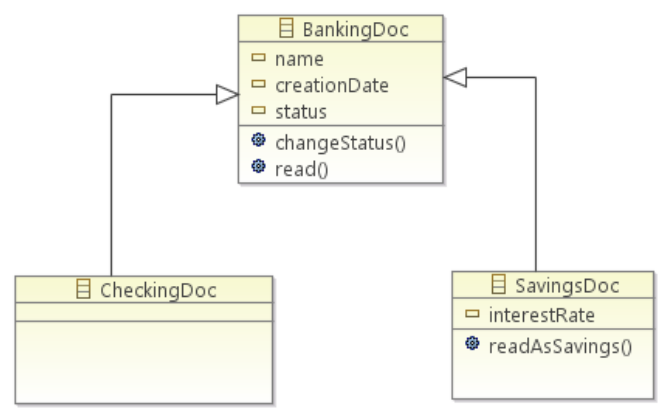

Figure 2. Inheritance in a business document repository

In OO programming, encapsulation refers to the encapsulation of properties and operations so that their interactions become clearly identified. To this end, the financial documents example of Figure 2 manifests encapsulation, as in the following examples:

- $\quad$ The BankingDoc parent only permits a changeStatus application to changes the status property and a read application to retrieve the status property of a banking document (i.e., any CheckingDoc and SavingsDoc type document). No other application access is allowed.

- A readAsSavings application can only be applied to a savings document. 
One needs to define a convention for identifying the properties a given application is allowed to operate on. For example, one possible convention is to use the application name changeStatus to imply that the application can only affect the status field/property. A different approach is to use the formal arguments in the UML diagram, such as changeExpense(Expenseltem e) of Figure 1, to mean that the changeExpense application can only modify the ExpenseItem field of the ExpenseReport document; we can further restrict the changeExpense application's access to the ExpenseItem field to read-only with the formal argument changeExpense(final ExpenseItem e).

Note that encapsulation in $\mathrm{OO}$ programming is a human modeling and design concept, not necessarily an implementation feature. A cloud user can benefit from encapsulation as an organizational and control feature, yet implementation can still keep data objects and applications separate.

In OO programming, subtype polymorphism, also universally called just polymorphism, is the ability of one type, $A$, to appear as and be used like another type, $B$. The same principle applies well to cloud documents. For example, consider a modification of the hierarchy of Figure 2 in which: (i) a banking document allows an edit application for the purpose of editing a banking document's name field only, and (ii) a savings document also allows an edit operation for the purpose of modifying the interest rate in addition to the operation permitted on the parent. If the end user applies an edit application to a checking document it will (using inheritance) apply it using (i), whereas an edit application to a savings document will apply it using (ii).

\subsection{Adaptation of Entity Relationships to Document Objects}

Classical OO modeling (of inheritance, encapsulation, and polymorphism) was merged with entity-relationship (ER) diagramming [3] to create the UML class diagram notation, such as in Figure 1. Some examples of relationships that must exist in an aggregate document that conforms to the schema of Figure 1 are:

- An expense report document must contain at least one expense element.

- A tax return document must be associated with twelve monthly statement documents.

- A business monthly statement can contain an expense report, but no more than one.

The next section addresses the question of whether unspecified relationships are permitted to exist in the actual cloud.

\section{Rule Enforcement}

Enforcement is the automated activity of enforcing the document organization schema on instances of actual documents and applications. The enforcement engine must check all relationships and properties in the actual document set for conformance with the schema. For example, per the schema introduced in Figure 1, the enforcement engine will check that all relationships specified in Figure 1 are conformed to by the document instances. Figure 1 specifies, for instance, that personal monthly bank statements (instances of PMonthlyStmt class, e.g., in PDF format) are associated with PTaxReturn object instances.

In addition, the enforcement engine must validate that instance documents on the cloud contain the properties specified by Figure 1. The enforcement engine must also restrict applications to operate only on certain data elements as specified by the schema; for example, changeExpense of the ExpenseReport class of Figure 1 is specified to operate only on instances of the ExpenseItem class (which is an element class, i.e., no verbatim ExpenseItem file needs to exist on the platform). According to the class diagram an ExpenseItem element must be a constituent element of an ExpenseReport document. Consequently changeExpense is prohibited from touching anything in any ExpenseReport file other than the Expenseltem instances, in contrast with contemporary applications that are free to do whatever they elect to do on their target documents. With most cloud implementations being based on prevailing contemporary operating systems, this kind of enforcement is not readily available. One possible solution however is for an event-based enforcement engine, discussed below, to allow objects "checkout" only when the request is made by an application that satisfies the criteria set by the schema.

\subsection{Schema Enforcement Scheduling}

Two enforcement scheduling approaches come to mind: periodic and event-based. A periodic enforcement engine traverses the cloud much like a crawler traverses the Internet. When it discovers objects it validates their structure (i.e., checks whether the objects' actual properties are in compliance with the schema), and traverses associations to yet another object on the cloud, for which it repeats the abovementioned procedure. This process amounts to graph traversal with additional validation of object-structure in each node.

The drawback of a periodic enforcement scheduling approach is that it does not detect violations at the time an end user of the system takes actions that violate a schema. For example, per the schema of Figure 1, if an end user removes a BMonthlyStmt document then all links in associated ExpenseReport documents break, yet the discovery of this anomaly will probably be delayed.

In an event-driven enforcement scheduling approach, a schema is validated whenever a relevant event occurs, such as a user modifying, moving, copying, or renaming a document file. One possible event-driven enforcement approach makes good use of CC as follows. Because CC serves as a gateway to the cloud, no object or application 
really exists unless the $\mathrm{CC}$ says so. Every meaningful modification to the elements registered in the $\mathrm{CC}$ environment is first detected by the $\mathrm{CC}$, allowing the enforcement engine to validate the integrity of the document set and its conformance to all schemas. If the validation fails, the action is disallowed; $\mathrm{CC}$ has the capability to rollback the state of the repository or even, in some implementations, block the violating action. We adapt the techniques from source control to implement these enforcement capabilities.

\subsection{Multi-Schema Considerations}

We envision environments in which a plurality of schemas are used and enforced. In such a context a single document type (e.g., class BTaxReturn of Figure 1) may be specified in more than one schema. The following are several interesting cases that could arise:

- Multiple inheritance. A document class $A$ might be specified to extend class $B$ in one schema and class $C$ in another. There is no ill-defined relationship here; the specified inheritance relationships will be enforced and used when their corresponding schema is enforced.

- Union of associations. A document class $A$ might have association rel_l with class $D$ specified by one schema and association rel_2 with class $E$ specified by another schema. If the two relationships are different (e.g., $D \neq E$ ) then, again, the situation does not induce an ill-defined relationship; the specified associations will be enforced and used when their corresponding schema is enforced. However, a conflict might occur if $D=E$ and the respective quantification does not agree, such as one having minOccurances $=5$ and the other having maxOccurances $=1$. The enforcement tool must prune out such contradictions when a schema is deployed.

- Unspecified associations. If a document object has a relationship that does not conform to any schema then rule enforcement must identify this as an error.

We also envision a situation in which hierarchical organizations will impose schemas in a hierarchical manner, much like state laws exist in addition to federal laws. To this end, an additional required feature is the ability of a schema to disallow certain associations. For example, an accounting firm might disallow direct associations between an expense report type document and a tax return document. Note that the XML approach of using the maxOccurences $=0$ constraint in an XML schema file is insufficient because it relates only to a specific association between two entities, not to all possible associations between that pair.

\subsection{Discovery}

When a document is added to the cloud, with either enforcement scheduling approach discussed earlier, a necessary precursor to schema enforcement is discovery, in which we map an object to one or more schemas. The following mappings are suggested to discover and map an instance document or application object to a schema:

(a) Document to schema classes; for example, per the schema of Figure 1, when a PDF file is added to the cloud we need to discover whether it is an instance of PMonthlyStmt, PTaxReturn, BMonthlyStmt, ExpenseReport, or BTaxReturn.

(b) Document to other documents according to the relationships described in the schema. For example, if the document discovered in (a) is a BMonthlyStmt class of Figure 1 then we need to discover its associated BTaxReturn document, if it exists.

(c) Document's parts, components, or elements to the properties of the class discovered in (a).

(d) Applications to the methods specified for its class in the schema. For example, if the class discovered in (a) is a BTaxReturn class document per the schema of Figure 1, then we need to discover the application that corresponds to getExpenses method of that class.

Note that this is a significant departure from the way operating systems typically treat applications. While traditionally the domain and co-domain of an application are mapped to one or more file objects, this mapping maps the domain and co-domain of the applications to particular components, parts, or elements of file objects as specified in the mapping (c) above. (Let $f: A \rightarrow B$ be a function from $A$ into $B$. The set $A$ is called the domain of the function $f$, and $B$ is called the co-domain of $f$.)

\subsection{Merging Documents}

Consider an often-discussed use case for the cloud in which a shared document $D$ is used by a group in the cloud. An individual user then downloads $D$, and edits it on his or her desktop (a situation that might arise when connectivity is disrupted), thereby creating version $D^{\prime}$. Later, this user wants to upload his or her version and merge it with the cloud version. This kind of use case is quite common in a software engineering setting in which users often work offline and then merge their code changes with the repository; contemporary source control tools provide ample tool support for such merge operations.

Merging documents that are serialized in binary or some other cryptic proprietary notation is clearly a nightmare. Fortunately, most contemporary applications use XML notation when serializing their documents. XML notation however does not solve the problem because the merge operation is not well defined. First, typical XML tags in most office documents are not semantic; rather, they identify editing information such as lines, paragraphs, and fonts, information that cannot be used for semantic merging. Secondly, consider an example where the documents $D$ and $D^{\prime}$ each contains a different element $\mathrm{el}$; should this case be pronounced as a conflict or should there be two el fields in the merged version? If $e l$ is the 
document owner's name, then most readers would agree that that field should be unique, that is, the two instances of $e l$ should be merged into one. However, if $e l$ is an expense item, then $D$ can contain a long list of expenses, i.e., both instances of $e l$ should be used in the merged document.

A schema such as in Figure 1 resolves these issues. If two ExpenseReport type documents are merged then the schema clearly specifies that an ExpenseReport can contain a plurality of ExpenseItem elements. Hence, the merge tool can simply create a union of all expenses in the merged ExpenseReport. We propose that the UML schema have a specification as to how to manage conflicts. For example, if the schema also indicates that the Date field of an ExpenseReport is unique and the dates in both merged documents do not agree, the merge tool will pick the most recent, or perhaps even the date of the merge, depending on the UML specification that pertains to a merge-conflict in that field.

\section{Cloud Control Environment}

Source control, also known as version control or software configuration control, deals with the management of changes to documents, programs, and other information stored as computer files. We incorporate the following aspects of source control into the CC environment.

- Multiuser environments. Software projects often have multiple developers. An object (e.g., a Java source code file) is often owned by a certain developer, but is often developed by multiple simultaneous users. We see a similar trend in the emerging cloud world; for example, multiple users owning and co-editing a shared Google Docs document for a potluck lunch organization.

- Ownership. Ownership of objects can change over time, such as when the original developer leaves the company, so much so that ownership manifestation is often reduced to a list of author comments on the top of a source code file. In other words, a sourcecontrolled document has a life of its own that persists beyond the lifespan of an individual employee or that of a certain machine. Source control is therefore the tool organizations use to locate objects. Document objects have a similar lifecycle, with CC providing the same lifecycle ownership support.

- $\quad$ Storage. The location of the source control repository can be independent of the location of an individual developer; in fact, source control systems cater well for a distributed development teamwork environment. Such flexibility is one of the benefits lay people see in the cloud approach.

- $\quad$ Online vs. offline usage. Engineers tend to use source control in two modes: one is directly connected to the repository, and the other is offline, with frequent commits. Many source control tools support both modes of operation. The second approach is useful when working offline, and as a means for developing code and not committing it until tested or approved by a higher authority. These use cases exist for many cloud users who are concerned about the availability of their data during network down times. One difference between source control and $\mathrm{CC}$ is the availability of applications, which is not supported by offline source control, yet we envision that offline cloud users will have access to some of their applications.

- Visualization. Visualization provides clear interactive indication as to the status of every object, where a marked object means that its local version differs from the repository version. Similar visualization will also be beneficial to cloud users.

The following are typical CC functions supported by a source control tool:

- Management of the physical storage of files in the repository and in the working folders of individual developers;

- Management of the historical record of the changes made on the files over time via version numbers, labels and tags;

- Checkin/checkout mechanisms to control concurrent access to the files; and

- Merge and branching mechanisms to allow developers to work on different parts of the software in parallel and merge their efforts later.

In addition to the above, any tool that supports cloud control should provide functionalities to:

- Specify and enforce the schematic rules on the relationships between the different document objects.

- Specify and enforce the relationship between the document objects and the different applications for processing the objects.

- Create, modify, search, retrieve and destroy the document objects and relationships.

\section{Related Work}

HyperText Markup Language (HTML) links provide a contemporary method for the specification of documentto-document and document-to-application links [4]. While HTML links provide a reasonable technique for the implementation of links, one that can be adopted by a realization of the ideas presented in this paper, HTML links do not support the OO principle of encapsulation.

The article "Build an Object-oriented File System in PHP" [5] describes a basic OO file system approach but does not address the specification and enforcement of the OO encapsulation principle, that is, it does not address the manner in which applications are strictly associated with property elements of specific document types. 
The patent "Object oriented file system in an object oriented operating system" [6] also describes a basic OO file system approach. The patent only addresses the OO principle of encapsulation in a limited manner whereby the only supported operations are standard operations (such as create, open, close, and property accessors) and their variations. The patent ignores the issue of enforcement of document-object relationships and application-to-object relationships because it does not cater for object-to-object relationships other than inheritance.

Moreover, OO operating systems, such as a Javabased OS, do not necessarily treat document objects and applications any differently than a C-based OS such as Unix. Specifically, the OO document object and application relationships specification and enforcement discussed in this paper are not addressed at all.

\section{Conclusion}

We believe that using a universally understood formalism for specifying and enforcing extended $\mathrm{OO}$ relationships, both between documents and between applications and documents, is superior to the prevailing ad hoc method of achieving similar business rules. Some of the benefits of the proposed approach are:

- Compositionality. A complex aggregate document can be adequately described by a large collection of small documents organized in a well-defined manner.

- Maintainability. An aggregate document is easily updated by replacing small constituent document elements while keeping the induced aggregate document intact. Also, an application can be changed (e.g., from Microsoft PowerPoint to the OpenOffice version) with assurance that the new version still operates on the intended fields of the intended object types.

- Correctness and Accuracy. When updating an aggregate document (e.g., by updating or replacing constituent document objects), automatic rule enforcement maintains the correct relationships within the aggregate. Applications are only permitted to operate on clearly specified elements of specified document types, thereby reducing the risk of inadvertent data corruption due to unintended consequences of execution of the application.

- Traceability. An integral part of the technology supporting $O O$ rule-enforcement is the ability to traverse associations.

- Sharing. An aggregate document schema can cross the boundaries of an individual user. Document objects can be shared by more than one aggregate document (as long as the application specifications in all schemas are free of conflicts).

- Portability. When constituent objects are relocated, the enforcement rule automatically validates that the constraints of all aggregate document schemas is still maintained.

There are many related topics this article has not addressed. For example, when a document type specifies an application (e.g. Foo.exe) that can operate on a specific property, how does an application on the cloud authenticate itself as Foo.exe? This and other questions will need to be asked and answered to gain momentum for adoption of cloud computing.

\section{Acknowledgment}

The research was supported in part by a grant from the Office of the Department of Defense Chief Information Officer. The views and conclusions contained herein are those of the authors and should not be interpreted as necessarily representing the official policies or endorsements, either expressed or implied, of the U.S. Government. The U.S. Government is authorized to reproduce and distribute reprints for Government purposes notwithstanding any copyright annotations thereon.

\section{References}

[1] B. Bruegge and A.H. Dutoit, Object-Oriented Software Engineering: Using UML, Patterns and Java, $3^{\text {rd }}$ ed., Prentice-Hall, 2009.

[2] D. Drusinsky, J.B. Michael, T.W. Otani, M. Shing, Putting Order Into the Cloud: Object-oriented UML-based Rule Enforcement for Document and Application Organization, Tech. Report NPS-CS-10-009, Naval Postgraduate School, Monterey, CA, September 2010.

[3] P.P. Chen, "The Entity-Relationship Model: Toward a Unified View of Data," ACM Trans. on Database Systems, vol.1, no.1, March 1976, pp. 1-36.

[4] World Wide Web Consortium (W3C), HTML 4.01 Specification, December 24, 1999. Accessed on 25 January 2011: http://www.w3.org/TR/html401/

[5] S. Scriven, "Build an Object-oriented File System in PHP," Internet.com, 25 October 2004. Accessed on 25 January 2011: http://www.devx.com/webdev/Article/22240/1954

[6] B.P. Atsatt, E.K. Nandkeshwar, M.J. Seilnacht, H.A. Thakkar, G.R. Turner, R.R. Webster, US 5758153: Object oriented file system in an object oriented operating system, Object Technology Licensing, May 1998. 